\title{
Las escuelas normales en Chile y Argentina (1821-1974). Un estudio comparado
}

\author{
Normal schools in Chile and Argentina (1821-1974). \\ A comparative study

\section{Camila Pérez Navarro $^{1}$ y Laura Graciela Rodriguez ${ }^{2}$}

\begin{abstract}
El presente artículo analiza, desde una perspectiva comparada, el proceso de creación y expansión de las escuelas normales en Chile y Argentina, desde principios del siglo XIX hasta la década de 1970. Con base en la revisión y análisis del contenido de diversas fuentes primarias documentales, dicho proceso se analiza a partir de tres ejes: las influencias extranjeras que recibió el normalismo en distintas épocas, el proceso de feminización del magisterio y las reformas más importantes que se implementaron. Pretendemos mostrar, que, en diferentes momentos, se recibieron aportes del sistema lancasteriano inglés, y de los modelos francés, norteamericano y alemán. En segundo término, señalaremos cómo, en ambos países, las autoridades pensaron en los inicios formar un magisterio masculino, pero al poco tiempo debieron cambiar la estrategia y abrir más normales para mujeres. Por último, señalaremos en qué sentido, las reformas de los años de 1940 en Argentina, y luego las de 1960 en las dos naciones, resultaron profundas y cambiaron la estructura del normalismo.
\end{abstract}

Resumen

Palabras clave: escuelas normales, influencias extranjeras, reformas, Argentina, Chile

\footnotetext{
${ }^{1}$ Instituto de Ciencias de la Educación, Universidad de O’Higgins. Correo electrónico: camila.perez@uoh.cl ${ }^{2}$ Consejo Nacional de Investigaciones Científicas y Tecnológicas (CONICET) de la Universidad Nacional de La Plata.Correo electrónico: lau.g.rodrig@gmail.com
} 
Abstract

The aim of this paper is analyze, from a comparative perspective, the process of creation and expansion of the normal schools in Chile and Argentina, from the early 19th century to the 1970s. Based on the review and analysis of the content of various primary documentary sources, the process is analyzed from three axes: the foreign influences that normalism received at different times, the process of feminization of the teaching profession, and the most important reforms that were implemented. We intend to show that, at different times, contributions were received from the English Lancasterian system, and from the French, American and German models. Secondly, we will point out how, in both countries, the authorities initially thought of forming a male teacher, but soon they had to change the strategy and open more Normal Schools for women. Finally, we will point out in what sense the reforms of the 1940s in Argentina, and then those of 1960 in the two nations, were profound and changed the structure of normalism.

\section{Keywords: Normal Schools, foreign influences, Argentina, Chile, history of education}

\section{Introducción}

Las escuelas normales fueron instituciones muy relevantes en los procesos de fundación y consolidación de los sistemas educativos modernos de América Latina. A lo largo del siglo XIX, tanto en Chile como en Argentina hubo distintos intentos de creación de escuelas normales que no prosperaron: el primer experimento por parte del gobierno nacional se dio en Chile en la década de 1820, y en Argentina, las primeras iniciativas fueron de gobiernos provinciales como el de Buenos Aires, donde se sucedieron aperturas y cierres entre las décadas de 1820 y 1870.

En ambos, fueron los gobiernos nacionales los que sentaron las bases del sistema normalista, a partir de 1842 en Chile y desde 1870 en Argentina. Al igual que en otros países, la creación de escuelas normales nacionales respondió a la necesidad de formar maestros y maestras que se ocupasen de alfabetizar a la mayoría de la población, moralizar a los sectores populares, transmitirles los principios básicos del higienismo y hacerlos parte de una misma comunidad nacional (Alliaud, 2007; Lionetti, 2007; Fiorucci, 2014; Cox y Gysling, 1990; Egaña, 2000; Egaña, Núñez y Salinas, 2003; Núñez, 2010).

Con el transcurrir del tiempo, el sistema se fue expandiendo y consolidando y, en ciertos aspectos, el balance resultaba muy positivo. Hacia mediados del siglo XX, en un informe realizado por un especialista internacional, se mencionaba cuáles eran los países que tenían el mayor porcentaje de maestros/as titulados en las normales, siendo Argentina y Chile -junto con Cuba y Uruguay- los que mejor estaban en el listado, mientras que Brasil, por 
ejemplo, presentaba un porcentaje de no titulados del $44 \%$ y Bolivia, del $82 \%$ (Revista de Educación, Nº100, 1959).

De todos modos, para esa misma época, la formación docente en ambos países comenzó a ser cuestionada porque se consideraba que no podía continuar en el nivel medio. Durante la década de 1960 se pasaron los cursos de magisterio al nivel post secundario en Chile y al nivel terciario en Argentina, donde en 1970 se abrió por primera vez la inscripción a los nuevos Institutos Superiores de Formación Docente. En Chile, la transformación continuó a la llegada del gobierno de facto de 1973, que decidió la clausura definitiva de las escuelas normales, dejando los estudios de magisterio en manos de las Universidades. A esa altura, en Chile las normales eran un total de 17 y en Argentina, alrededor de 174.

Con base en lo expuesto hasta aquí, en este artículo analizaremos el proceso de creación y expansión de escuelas normales en Chile y Argentina, desde principios del siglo XIX hasta la década de 1970, a partir de tres ejes: las influencias extranjeras que recibió el normalismo en distintas épocas, el proceso de feminización del magisterio, y las reformas más importantes que se implementaron. Pretendemos mostrar, en relación con el primer punto, que en diferentes momentos se recibieron aportes del sistema lancasteriano inglés, y de los modelos francés, norteamericano y alemán. Sobre la feminización del magisterio, plantearemos que, en los dos países, las máximas autoridades apostaron en los inicios a crear normales de varones y formar un magisterio masculino, pero al poco tiempo debieron cambiar la estrategia, dado que los varones, ante los bajos salarios que se pagaban, terminaban dejando los estudios o la profesión. Comenzaron entonces a privilegiar la fundación de normales femeninas, sosteniendo que ellas estaban "naturalmente" destinadas para la enseñanza de los/as niños/as más pequeños/as, y estarían más dispuestas que ellos, a percibir sueldos menores ante la ausencia de otras perspectivas. En tercer término, señalaremos en qué sentido las reformas de los años de 1940 en Argentina y luego las de 1960 en ambos países -estas últimas bajo la influencia de los organismos internacionales y la necesidad de "modernizar" el sistema educativo- resultaron profundas y cambiaron la estructura del normalismo.

Ahora bien, este artículo está dividido en cinco apartados: en el primero estudiaremos el sistema lancasteriano como el primer modelo de formación de docentes que influyó en Chile y Argentina durante la década de 1820. En el siguiente apartado, ahondaremos en el proceso de fundación de escuelas normales y el rol de Domingo Faustino Sarmiento en ambos países; luego, examinaremos la influencia educativa proveniente de Alemania para el caso de Chile y de Norteamérica en Argentina. En el cuarto apartado, indicaremos las políticas relativas a la formación normalista adoptadas entre 1920 y 1940 y, finalmente, analizaremos la influencia de los organismos internacionales a partir de la segunda mitad del siglo XX. 


\section{El sistema lancasteriano y la formación de preceptores/as (ca. 1820 - ca. 1840)}

\subsection{El sistema lancasteriano en Argentina}

Los gobiernos independentistas de Argentina y Chile, en la década de 1820, decidieron organizar cursos con el propósito de implementar el sistema lancasteriano o de enseñanza mutua. Este método, creado por el profesor inglés Joseph Lancaster a fines del siglo XVIII, "parecía adecuado para ilustrar a los ciudadanos de las repúblicas recién constituidas" (Rojas, 2010: 60). En 1818 la Sociedad Lancasteriana de Londres designó a uno de sus miembros, Diego Thompson, para que recorriera los países de América (llegó a estar en los actuales territorios de Colombia, Chile, Perú, Uruguay, entre otros) y publicitara las excelencias del método (Ramos, 1910; Salvadores, 1941). Este tuvo un éxito casi inmediato porque a los Estados les resultaba más barato que los métodos tradicionales: en teoría, un maestro podía enseñar en un mismo salón a cientos de niños, solo con la ayuda de los "monitores", quienes eran los alumnos de la misma clase que estaban un poco más avanzados.

Thompson arribó el 6 de octubre de 1818 a Buenos Aires y formó una Sociedad apoyado por el Presbítero Bartolomé Muñoz. A poco de llegar, fundó y sostuvo 16 escuelas: 8 en la ciudad y 8 en la campaña. En mayo de 1821 el Cabildo de Buenos Aires resolvió adoptar su sistema y financiar las escuelas, apoyando decididamente "al célebre método Lancaster" (Ramos, 1910: 339). Hubo escuelas lancasterianas en algunas ciudades de Entre Ríos, Santa Fe, Jujuy, Tucumán y Salta (Salvadores, 1941: 95). Cuando los niños eran muchos, el sistema solo podía llevarse a cabo manteniendo una disciplina férrea que sostuviera cierto orden en la clase.

En 1821 el gobernador de la provincia de Buenos Aires, Martín Rodríguez, creó la Universidad de Buenos Aires y en 1822 se pusieron las escuelas elementales bajo su dependencia. Hasta ese momento, estos pocos establecimientos sostenidos por el Estado eran solo para los varones. Las primeras escuelas de niñas fueron creadas a partir de 1823 por la Sociedad de Beneficencia, bajo el sistema lancasteriano. La Sociedad estaba conformada por un grupo de mujeres de la élite porteña, cuya presidenta era María Sánchez de Mendeville. El ministro Rivadavia le otorgó a la institución importantes subsidios para que se hicieran cargo de varias funciones, entre ellas, la dirección e inspección de las mencionadas escuelas de niñas. En 1824 la Sociedad creó la primera Normal de mujeres en la ciudad de Buenos Aires que tuvo 93 alumnas (Portnoy, 1937: 78). Las encargadas de la Sociedad fueron creando más escuelas primarias y ocho años después, Mendeville informaba que las maestras formadas en la Normal tenían a su cargo siete escuelas de las ocho que había en la ciudad y tres de las seis de la campaña (Salvadores, 1941: 142). Sarmiento elogió enfáticamente a la Sociedad 
por su labor educativa a través de la cual "las mujeres de todas las clases gozaban de una educación regular y sostenida por el erario" (Sarmiento, 1849: 107).

En 1826, siendo presidente Rivadavia y gobernador de la provincia de Buenos Aires Juan Gregorio Las Heras, promovieron la creación de una Normal de preceptores varones con la dirección del español Pedro Baladía, siguiendo el sistema lancasteriano y un plan de dos años. Baladía obligó a los preceptores que estaban trabajando, a concurrir a las clases de la Normal para recibir sus lecciones, lo que hizo que casi no pudiese funcionar a causa de los conflictos que se generaron con los maestros (Newland, 1992).

Con la llegada de Juan M. de Rosas al gobierno, la Sociedad de Beneficencia y las escuelas públicas en general, se vieron perjudicadas porque se impuso el arancelamiento y en 1838 se las dejó de subvencionar. La Sociedad, si bien debió cerrar escuelas, entre ellas la Normal, logró que ciertos privados financiaran algunas otras.

\subsection{El sistema lancasteriano en Chile}

En el caso chileno, la aplicación del método lancasteriano tuvo una trayectoria más breve. En febrero de 1819 el gobierno de Bernardo O’Higgins y el Senado determinaron la aplicación del reglamento de instrucción primaria que Manuel Belgrano había puesto en práctica en el Virreinato del Río de La Plata (Amunátegui, 1895). Este reglamento creaba la figura de protector general, regulaba el ejercicio de la docencia, establecía las asignaturas del plan de estudio y los principios y normas de conducta a inculcar en los estudiantes, y establecía los exámenes públicos. Sin embargo, se carecía de dos elementos indispensables: faltaban preceptores idóneos y fondos suficientes (Amunátegui, 1895). En este contexto, llegaron las primeras noticias del sistema de Lancaster. Se contrató al pedagogo inglés Diego Thompson para que implantara el sistema en Chile de la misma forma que lo había hecho en el virreinato del Río de la Plata (Serrano, 1993).

En septiembre de 1821 fue creada una escuela lancasteriana en la capilla de la Universidad de San Felipe, mientras que en noviembre de ese mismo año se decretó la asistencia de todos los maestros de primeras letras a la primera Escuela Normal de Enseñanza Mutua (Rojas Flores, 2010). Su fundación respondió al propósito de instruir en el nuevo sistema de enseñanza a todos los maestros (Amunátegui, 1895) y así, uniformar los métodos de enseñanza. Esta escuela logró reunir 140 estudiantes en 1822 (Serrano, 1993) y había sido "creada para que sirviese de norma" (Mancilla, 2005: 25). Además, en enero de 1822 se fundó una Sociedad Lancasteriana con el propósito de propagar el sistema de la enseñanza mutua (Munizaga, 1990).

Puesto que el contrato de Thompson era acotado, el gobierno buscó un reemplazante, eligiendo a Anthony Eaton, quien llegó a Chile a fines de 1821 y se dedicó a preparar preceptores (Rojas, 2010). Si bien continuó funcionando a lo largo de la década, hacia finales del 
decenio, el sistema Lancaster comenzó a recibir críticas por distintas razones. Además, Eaton enfermó y regresó a Inglaterra, abandonándose este nuevo sistema de enseñanza (Amunátegui, 1895). Estas críticas estaban relacionadas, principalmente, con el uso de ciertos textos de enseñanza o la falta de recursos para continuar con su implementación (Serrano, 1993).

\section{Influencias francesas y norteamericanas \\ (ca. 1840 - ca. 1870)}

\subsection{La fundación de la primera Normal de varones (1842) y de mujeres en Chile (1854)}

La década de 1840 es clave en el proceso de fundación del sistema educativo chileno (Egaña, 1994 y 2000). El educador argentino Domingo Faustino Sarmiento, quien se encontraba exiliado en Chile debido a su oposición al gobierno de Juan Manuel de Rosas, impulsó la creación de una Escuela Normal masculina de Preceptores.

De acuerdo con su decreto de creación, publicado en enero de 1842, la finalidad de esta institución era preparar a los maestros que dirigirían las escuelas primarias "en toda la extensión de la República" y, así, propender a "la mejora de las costumbres y todo progreso intelectual, sólido y verdadero". El principal objetivo de la Normal fue formar "maestros idóneos y de reconocida moralidad, mediante métodos fáciles, claros y uniformes". Para ingresar, los postulantes debían tener una edad mínima de 18 años, instrucción regular en leer y escribir, acreditar buena conducta y "pertenecer a una familia honrada y juiciosa". El currículum normalista incluía los siguientes ramos: "leer y escribir a la perfección, y un conocimiento completo de los métodos de enseñanza mutua y simultánea; dogma y moral religiosa; aritmética comercial; gramática y ortografía castellana; geografía descriptiva; dibujo lineal; nociones generales de historia y particulares de la de Chile". Se estableció que inicialmente serían 28 alumnos, quienes recibirían "cien pesos anuales para los gastos de su manutención y vestuario" (Chile, 1842: 3).

A fines de 1845, las autoridades determinaron que la Escuela Normal comenzaría a funcionar con régimen de internado, puesto "que la experiencia ha demostrado que la circunstancia de ser externos los alumnos se opone a su mayor aprovechamiento y dificulta la vigilancia que sobre su conducta y moralidad deben ejercer los superiores del establecimiento”. Además, como se señala en el Decreto, esta medida permitiría que concurrieran a la Normal "jóvenes de las varias provincias del Estado, como lo exige el bien de la instrucción primaria" (Chile, 1845: 2). 
Como sostiene el historiador Juan Pablo Conejeros (1999: 21), en un contexto en que los ideales ilustrados franceses marcaban "el sello del nuevo pensamiento educacional", la Escuela Normal de Preceptores fue modelada según la École Normale francesa (Ávalos, 2003). Sarmiento fue designado como director (Ministerio de Educación Pública, 1945), cargo en el que se mantuvo hasta 1845, momento en que se le encarga "un viaje por países europeos que le permitiera ampliar sus horizontes en materia de Educación y Cultura" (Conejeros, 1999: 25). En palabras de Núñez, "si no fuera por la presencia de Domingo Faustino Sarmiento como director y único profesor, la primera normal pudo haber parecido una institución intrascendente" (2010: 135). Durante la década de 1840, la Normal de Preceptores "fue poco más que una escuela primaria en que se enseñaba a jóvenes de los sectores pobres, difícilmente reclutados entre quienes apenas sabían leer y escribir" (Núñez, 2010: 135).

Años más tarde, frente a la necesidad de confiar la instrucción primaria de mujeres a "maestras idóneas y de conocida moralidad" en 1854 fue creada la Escuela Normal de Preceptoras de Santiago, la cual funcionó con un régimen de internado. Como señalaba su decreto de fundación, la Normal formaba a las alumnas en "lectura y escritura, dándose al mismo tiempo un conocimiento completo de los métodos de enseñanza mutua y simultánea, dogma y moral religiosa, gramática castellana, aritmética, geografía, dibujo e historia, costura, bordado y otros trabajos de aguja”. Las postulantes debían cumplir con los mismos requisitos de ingreso que en la Normal de Preceptores, salvo con la exigencia relativa a la edad. En sus primeros años de funcionamiento, esta institución fue gestionada por la congregación francesa del Sagrado Corazón (Peña, 2000). Esto posibilitó que las religiosas tuvieran la "libertad para decidir la forma de enseñanza, los ramos de estudio y el reglamento interno" (Orellana, 2007: 41). Como sostiene el investigador Fredy Soto Roa, es probable que "la garantía de una formación casi conventual [tuviera], en ese momento, una influencia positiva", aceptándose "la incorporación de la mujer a una profesión rentada" (2013: 400). La gestión de las religiosas fue evaluada negativamente por el ministro de Justicia, Culto e Instrucción Pública a fines de la década de 1850, quien "se quejaba que la Normal no producía resultados satisfactorios (...) [Puesto que] en diez años solo egresaron 63 normalistas" (Soto Roa, 2013: 401).

De acuerdo con lo señalado por la destacada educadora Amanda Labarca (1939), entre las décadas de 1840 y 1880 la influencia francesa dominó sin contrapeso en la historia educativa chilena. Esto se evidencia en la "presencia de diversas congregaciones religiosas de origen francés consagradas a la enseñanza" (Conejeros, 2015: 39). Francia era vista por los chilenos como una garantía de progreso y civilización. Por este motivo, el mismo Sarmiento fue enviado por el gobierno chileno a Francia en 1845, a examinar los sistemas públicos de educación primaria (Sanhueza, 2013). 


\subsection{Sarmiento y la influencia norteamericana en Argentina}

En 1852, en la provincia de Buenos Aires se restableció el subsidio estatal a la Sociedad de Beneficencia y se declaró la gratuidad escolar en las escuelas públicas. Así también, se propuso la fundación de una nueva Normal de varones, que no prosperó. Buscaba seguir, igual que en Chile, el modelo francés de la École Normale Supérieure, con un plan de estudios de 20 materias y una duración de cinco años (Salvadores, 1941). Se nombró regente a Marcos Sastre y administrador a Germán Frers, pero la Normal no alcanzó a constituirse debido a los enfrentamientos políticos que derribaron al gobierno provisional (Salvadores, 1941).

En abril de 1855 la Sociedad de Beneficencia volvió a abrir una Normal para mujeres, con subvención estatal, siendo su primer director Frers. A mediados de 1873 la Normal se puso bajo la dirección de la maestra norteamericana Inés E. de Trégent, contratada por el gobierno argentino, quien formó "un cuerpo docente de primer orden" donde se daban física, química, matemáticas, historia natural, geografía, historia, cosmografía y astronomía, entre otras asignaturas, adoptando los métodos y sistemas de enseñanza de los Estados Unidos (Fernández, 1903: 105). Dicha escuela normal funcionó durante 21 años hasta 1876, y egresó de ella la mayor parte de las maestras de su tiempo.

Hacia 1865 el gobernador de la provincia de Buenos Aires creó otra Normal de varones, nombrándose a Marcos Sastre director -quien renunció al poco tiempo- y vice a Enrique M. de Santa Olalla (Ramos, 1910). La Normal solo produjo siete preceptores en seis años de funcionamiento regular y fue suprimida en 1871 porque no había relación entre el elevado costo y sus resultados (Salvadores, 1941).

Siendo jefe del Departamento de Escuelas de Buenos Aires, Sarmiento presentó sendos informes en los años de 1850, donde argumentaba que, en su experiencia, había visto que las mujeres, por su condición de madres o futuras madres, estaban mejor preparadas para el magisterio que los varones y que, al no tener las mismas oportunidades laborales, aceptaban dedicarse a ser maestras, profesión donde el Estado, igual que en países como Estados Unidos, les pagaba sueldos más bajos. Decía Sarmiento que los maestros en Buenos Aires costaban en ese momento " 800 pesos al mes, mientras que las maestras están bien pagadas con 500 pesos y aún costarían menos para escuelas menos numerosas (...) [podrían llegar a] 200 pesos mensuales de retribución" (Sarmiento, 1954: 28).

Luego de estas fundaciones poco exitosas de normales de parte de la provincia, la nación comenzó a invertir por primera vez en estas instituciones. Siendo presidente Sarmiento, en julio de 1869 se crearon en algunas jurisdicciones, cursos normales para varones, anexos a los Colegios Nacionales (masculinos), que con el tiempo se fueron convirtiendo en normales completas. Finalmente, en octubre de 1869 el Congreso sancionó una Ley de autorización al Poder Ejecutivo para verificar los gastos de creación de dos normales, una en Paraná y otra 
en Tucumán, destinadas a formar Preceptores/as de instrucción primaria. Seguidamente, el presidente y su ministro Nicolás Avellaneda decretaron el 13 de junio de 1870 la instalación de una Normal en la ciudad de Paraná, que comenzó a funcionar en 1871. A iniciativa de Sarmiento, el gobierno argentino contrató -entre 1869 y 1898 - alrededor de 65 maestros y maestras oriundos de Estados Unidos (ellas eran mayoría) para que se hicieran cargo de la dirección de las normales de mujeres.

La mencionada Normal de Paraná, inicialmente fue de varones y al poco tiempo se hizo mixta, aunque mantuvo una matrícula mayoritariamente masculina durante más de una década. Tenía un plan de estudios de cuatro años de duración al que se sumó el del Profesorado normal, con un año más. En 1875 se inauguró la Normal de Tucumán que se inició mixta pero luego fue de varones, con un plan de dos años que pasó a tres al poco tiempo. Un año antes, en 1874 la provincia de Buenos Aires había inaugurado dos normales, una de mujeres y otra de varones, que en 1881 se nacionalizaron.

Desde los inicios, todas las normales formaron parte del nivel medio y se organizaron con una primaria anexa o Escuela de Aplicación de seis grados para que los/a maestros/as pudieran realizar sus prácticas, por lo que se les exigía a los aspirantes haber cursado hasta sexto grado. Las niñas debían ingresar con 14 años y los varones con 16. Los que no tenían el certificado de la primaria completa, tenían que rendir un examen no eliminatorio de nivelación.

Estas normales no tuvieron internado como en Chile, y el Estado nacional -y en menor medida los Estados provinciales y municipales- destinaron recursos para financiar becas a los/as alumnos/as maestros/as, logrando una amplia cobertura. Se consideraba que los/as alumnos/as de las normales eran empleados estatales desde el primer año que recibían la beca y se les hacía firmar a sus tutores un documento donde se los obligaba a ejercer la docencia durante un cierto período, después de recibidos.

En 1875, siendo presidente Nicolás Avellaneda y ministro Onésimo Leguizamón, se aprobó una Ley para fundar y sostener catorce normales de mujeres en cada una de las capitales de provincia, con un plan de tres años de duración. Un tiempo después, se fueron creando en esas ciudades, normales de varones. En referencia a la influencia norteamericana, un alto funcionario dijo en un acto público sobre la Normal de Paraná, que "fue una escuela de Boston trasplantada en las soledades de América del Sud", ya que "fue norteamericana por sus directores, por sus regentes, por su mobiliario y útiles, por la traducción de sus libros ingleses, por su táctica escolar militarizada y uniforme, por sus procedimientos y doctrinas" (El Monitor, 1915: 14).

Por esta vía norteamericana llegó también cierta influencia alemana: en 1884 comenzó a funcionar en la Normal de Paraná el primer Jardín de Infantes o Kindergarten, bajo la dirección de la norteamericana Sara C. de Eccleston, quien había estudiado en su país las teorías del pedagogo alemán Frederick Fröebel. Orientados por Eccleston, las autoridades 
argentinas encargaron la compra en Estados Unidos de todo el mobiliario y los útiles de enseñanza froebelianos.

\section{La feminización del magisterio en Chile y Argentina (ca. 1870 - ca. 1920)}

\subsection{El 'Embrujamiento alemán' y el proceso de feminización del magisterio en Chile}

La creación de dos nuevas escuelas normales femeninas en 1871 y 1874 en provincia constituyeron un antecedente a los procesos de feminización y profesionalización del magisterio en Chile. Su fundación respondió a la necesidad de instruir a las niñas de los sectores populares, junto con la consideración de que la docencia constituía una fuente de trabajo adecuada para el sexo femenino. Además, las mayores posibilidades de los hombres para conseguir una ocupación mejor remunerada y, por consiguiente, el abandono del ejercicio docente, impulsaron la creación de escuelas normales para preceptoras. Entre 1854 y 1909 funcionaron 12 escuelas normales para mujeres (aunque tres de ellas fueron cerradas a los pocos años de funcionamiento), mientras que solo se fundaron 6 escuelas normales para hombres. Esto significó que, desde mediados de la década de 1870, el número de preceptoras superara a la cantidad de hombres egresados. Para 1910, más del 70\% de los preceptores eran mujeres (Oficina Central de Estadística, 1910).

Con la creación de estas escuelas normales se introdujeron modificaciones en el plan de estudios y se adoptaron nuevos métodos de enseñanza. Esto evidenció un "concepto más avanzado, en orden de la preparación científica, literaria, pedagógica y metódica" (Muñoz, 1918: 142). Estos hechos permitieron el inicio de una nueva etapa en la formación normalista, caracterizada por el predominio de la pedagogía alemana. Además, los viajes que realizan intelectuales chilenos a Alemania marcan "el punto de partida de un intenso, profundo y complejo proceso de transferencia cultural que experimentará el país entre 1880 y 1910" (Conejeros, 2015: 43).

Uno de los intelectuales comisionados por el gobierno chileno fue José Abelardo Núñez. Luego de más de tres años y medio de permanencia en el extranjero, Núñez regresó al país. En su informe, titulado Organización de escuelas normales, Núñez relató su paso por las normales de Nueva York, Massachusetts, Filadelfia y Ohio y Alemania. En este último país, Núñez se sorprendió "el alto nivel de desarrollo y profundidad con que allí se abordaba la ciencia pedagógica" (Conejeros, 2015: 50). Por este motivo, el comisionado además incluyó en su escrito un anexo específico sobre Alemania, relativo al plan de estudios e información 
relativa a los exámenes. Con base en la información recopilada, Núñez sostenía que, desde la fundación de la Escuela Normal de Preceptores en 1842, la enseñanza normal "ha decaído, ha permanecido estacionaria e ignorante de los progresos alcanzados por la educación moderna en ese período de tiempo" (Núñez, 1883: 302).

En este contexto, desde 1883 se inició un proceso de reforma educativa de inspiración alemana. El informe entregado por Núñez "fue gravitante en la reorganización de las escuelas normales, así como la actualización del ideario curricular y modernización de las instituciones formadoras de preceptores y preceptoras" (Mansilla, 2018: 198). Esto puede evidenciarse en una ley promulgada el 11 de octubre de 1883, la cual permitió al presidente de la República, entre otras cosas, "contratar en el extranjero profesores de uno y otro sexo (...) para el servicio de las escuelas normales y primarias superiores del país" e "invertir hasta quince mil pesos en las pensiones y gastos de viajes de los alumnos y maestros de la Escuela Normal de Preceptores que se envíen a Europa o Estados Unidos, para desempeñar a su vuelta el cargo de preceptores de escuelas primarias" (Labarca, 1939: 183).

Este plan de estudios tuvo un carácter más profesionalizante, en la medida que integró la enseñanza de disciplinas con la formación en pedagogía teórica y práctica. Con la aplicación de este nuevo plan de estudios, la duración de la formación del preceptorado nuevamente se extendería, esta vez, a cinco años. La decisión de aumentar la duración de la formación normalista respondió a la necesidad de preparar a los estudiantes y suplir las deficiencias que presentara el alumno respecto de sus estudios primarios.

Nuevos requisitos de ingreso y promoción fueron establecidos por las autoridades en 1899, año en que se publicó el Reglamento General para las escuelas normales. Este decreto instruyó por primera vez, como era en Argentina, la obligación de contar con una escuela pública anexa (con el objetivo de que sus alumnos practicaran el ejercicio del preceptorado), además de la aceptación únicamente de postulantes que acreditaran haber cursado los estudios correspondientes al sexto año de escuela primaria (por lo tanto, la educación normal pasaría a ser ofrecida en nivel de enseñanza secundaria) y con un mínimo de 14 años de edad. Asimismo, se estableció como requisito la presentación por parte de los aspirantes de un certificado de conducta entregado por el director del último establecimiento que haya frecuentado el joven, un certificado de recomendación por parte de dos personas conocidas que acreditara la pertenencia a una familia honorable, y un certificado de constitución física y de buen estado de salud.

Un nuevo plan de estudios se aplicó en 1913 en las escuelas normales. Éste innovó en el establecimiento de una rígida distribución horaria de las asignaturas (especificaba las horas semanales de cada materia) y en el aumento de la formación práctica. 


\subsection{La feminización del magisterio en Argentina}

Hemos visto que tanto en Chile como en Argentina las primeras escuelas normales fueron, mayoritariamente, de un solo sexo, aunque en el segundo país, las normales mixtas comenzaron a fundarse tempranamente a partir de 1886 (la primera fue la de Paraná). En otro trabajo hemos observado que los primeros planes de estudio que se diseñaron entre 1876 y 1887 fueron distintos según el sexo, ya que tenían menos carga horaria para las mujeres en materias como Aritmética, Álgebra, Fisiología, Geometría, Física, Trigonometría, Agrimensura, Química, Lógica y Astronomía, porque en ese horario ellas debían cursar Labores y Economía Doméstica (Rodríguez, 2021). Luego de varios eventos, los planes finalmente se igualaron a partir de 1903, conservando solo las materias prácticas diferenciadas por sexo (Rodríguez, 2021). Del mismo modo, los salarios de las maestras de la ciudad de Buenos Aires fueron más bajos hasta principios del siglo XX, que se igualaron completamente, aunque continuaron siendo menores en otras provincias (Rodríguez, 2021).

Con respecto al proceso de feminización del magisterio, indicamos que este tuvo tres aspectos (Rodríguez, 2021)3. En primer lugar, vimos que dicha feminización se dio en la provincia de Buenos Aires, antes que en el resto del país gracias a que Sarmiento estimuló la presencia de las mujeres y hacia 1870, todas las escuelas infantiles mixtas (hasta los ocho años) y de niñas, tenían maestras a cargo, llegando a haber más cantidad de maestras que de maestros. En segundo lugar, si al comienzo del normalismo en las distintas provincias, los varones representaban una cantidad importante, al poco tiempo las estadísticas empezaron a mostrar que los inscriptos eran cada vez menos y los que egresaban, abandonaban la profesión rápidamente. Debido a esto y en el contexto de una profunda crisis económica, en el año 1900 se dio a conocer un Decreto que ordenó la fusión de las doce normales de Maestros en los Colegios Nacionales. En los hechos, la medida significó el cierre definitivo de 12 de 13 cursos de magisterio masculinos que existían hasta ese momento, lo que reforzó la feminización de la profesión. La tercera medida que se tomó en este sentido fue la fundación entre 1874 y 1914 en la ciudad de Buenos Aires, de 11 normales, de las cuales 10 fueron solo de mujeres, lo que aumentó exponencialmente la cantidad de egresadas. Esta gran cantidad de normales contrastaba abiertamente con la escasez de otro tipo de escuelas para mujeres. Por ejemplo, en la década de 1920 se habían creado solo dos Liceos de Señoritas en la ciudad. Producto directo de estas medidas, hacia la década de 1910 se comenzaron a escuchar numerosas voces que alertaban acerca "del exceso" de maestras en la ciudad de Buenos Aires que no conseguían trabajo. En síntesis, un observador de la época advertía que si en el quinquenio de 1874 a 1878 inclusive, los maestros a nivel nacional representaban un 85\% y

${ }^{3}$ Todo este párrafo está basado en Rodríguez (2021). 
las maestras un 15\%; en 1913, a 40 años de distancia, los términos se habían invertido y el $85 \%$ correspondía a las mujeres, mientras el $15 \%$ a los varones.

\section{Diferenciación entre escuelas normales rurales y urbanas, y reformas a la formación normalista (ca. 1920 - ca. 1940)}

\subsection{Reformas en la escuela normal chilena}

Durante la década de 1920, el sistema educativo chileno fue objeto de reformas. La aprobación de la Ley de Educación Primaria Obligatoria en agosto de 1920 consagró, al menos simbólicamente, el derecho a la educación primaria (Serrano, Ponce de León y Rengifo, 2013). Sin embargo, debido a sus limitados resultados y su falta de aplicabilidad en algunos ámbitos, diversos actores demandaron la implementación de una reforma educativa estructural en los años siguientes a su promulgación (Pérez Navarro, 2020).

En 1927, durante la dictadura del militar Carlos Ibáñez del Campo, fue puesta en marcha una reforma general del sistema educativo. Liderada por la Asociación General de Profesores (AGP), la reforma estableció la supresión de las antiguas normales (Chile, 1927) y, en su reemplazo, determinó la creación de escuelas de profesores primarios y escuelas de profesores secundarios (Chile, 1928), con el propósito de eliminar las diferencias formativas y salariales entre el profesorado primario y secundario (Reyes, 2014; Pérez Navarro, 2020). Pero, en agosto de 1928, se produjo un conflicto en las normales femeninas de Chillán y Angol que evidenció la oposición de sectores tradicionalistas del magisterio a aplicar nuevas prácticas pedagógicas y del Ministerio de Hacienda a desembolsar recursos (Reyes, 2010; Vial, 1996). Ibáñez suspendió la reforma e inició una contrarreforma, la cual mantuvo las ideas pedagógicas de la Escuela Nueva en los procesos de formulación de política educativa (Pérez Navarro, 2020), pero sin llevar a cabo un proceso de reestructuración administrativa como la impulsada por la AGP. En este contexto, Ibáñez promulgó la Ley de Enseñanza Normal (Chile, 1929). Bajo esta ley fueron creadas las normales rurales, las cuales estarían ubicadas en zonas de importancia agrícola. En tanto, las normales urbanas estarían destinadas a la formación del profesorado urbano y de cursos infantiles o kindergarten (Chile, 1929).

El Reglamento de admisión de alumnos de las normales, publicado en febrero de 1929, estableció nuevos requisitos de ingreso: para ser alumno de las normales rurales se exigió haber terminado el $6^{\circ}$ año de educación primaria y tener entre 14 y 18 años, mientras que para ingresar a las normales urbanas se debía completar el $3^{\circ}$ año de educación secundaria, y tener entre 15 y 20 años. Se mantuvieron los requisitos relativos al certificado de buena salud y de pertenencia a una familia honorable. Respecto a los exámenes de admisión, se 
conservó la prueba de conocimientos, mientras que se incluyó una prueba de inteligencia y madurez mental. Por otro lado, es importante señalar que el nuevo plan de estudios estableció como examen de graduación, la realización de una práctica en aula de un mes de duración, y la realización de una memoria de investigación sobre una temática educacional.

Conviene explicar un cambio introducido en 1929 en los procesos de promoción y graduación de alumnos normalistas. De acuerdo con el párrafo IV de la Ley de Enseñanza Normal, 5.100, al término de los estudios, la Normal otorgaría una licencia de profesor practicante, para enseñar en una escuela urbana o rural. Esta licencia tendría una duración de un año. Si al finalizar este período el profesor practicante demostraba buena conducta y cumplimiento de sus deberes, se le conferiría el título de profesor normalista. Si el profesor practicante no lograba cumplir con las expectativas, se le otorgaría la licencia provisoria por un año más. El fracaso del segundo año lo inhabilitaría para seguir trabajando como profesor.

\subsection{Argentina: la reforma Rothe de 1941}

En relación con las escuelas destinadas a formar un magisterio rural, a partir de 1910 se comenzaron a crear unas normales ubicadas en localidades de pocos habitantes, denominadas "de Preceptores" que tenían planes de estudio de dos años, pero sin orientación agraria. Debido a la crisis económica de 1930 y por su escasa orientación específica, fueron cerradas. Desde el año 1932, se fueron reabriendo estas normales y fundando nuevas, que tenían planes de estudio de cuatro años iguales a las "urbanas", y materias agrarias, resultando unas 18 en total (Rodríguez, 2020).

Igual que sucedió en otros países, entre los años de 1920 y 1940, hubo distintas experiencias en diferentes normales, de implementación de versiones propias de la Escuela Nueva, pero que fueron abandonándose, debido a que las máximas autoridades no invirtieron en recursos e infraestructura para sostenerlas.

En referencia a las reformas, de todas las que hubo, la más trascendente fue la que llevó a cabo el ministro Guillermo Rothe en 1941, por medio del Decreto 101107. El Ilamado "plan Rothe" estableció la división de los estudios del bachillerato y del magisterio en dos ciclos: el primero era común para ambas ramas de la enseñanza media y el otro diferenciado de acuerdo con las finalidades propias de cada una. En este sentido, fue parecida a la que se hizo en Chile en 1929 con las normales urbanas. Esta organización, según se aclaraba en la norma, permitió uniformar la preparación básica de los futuros bachilleres y maestros, para evitar que los/as estudiantes se viesen obligados a definir prematuramente su orientación hacia unos u otros estudios. Con esta reforma, además, los/as normalistas quedaron habilitados a ingresar a la universidad, aunque antes de ese año podían estudiar, por ejemplo, solo en los profesorados de Filosofía y Letras de la Universidad de Buenos Aires y de la Facultad 
de Humanidades y Ciencias de la Educación de la Universidad de La Plata y en determinadas carreras de esta última universidad. A partir de este Decreto de 1941, comenzaron a crearse tanto normales nuevas como "Ciclos de Magisterio" -solo los dos últimos años- anexos en su mayoría a los Colegios Nacionales, y en menor medida, a las Escuelas Nacionales de Comercio. Sin dudas, estas últimas fundaciones resultaban mucho menos costosas, ya que significaban conformar solo los cuartos y quintos años de la formación específica. Sin embargo, importantes normalistas criticaron esta reforma, afirmando que terminó desvirtuando "el espíritu original" de las normales y advirtiendo que la formación de dos años era claramente insuficiente (Rodríguez, 2019). Cabe añadir que, para esa época, la mayoría de las normales eran mixtas, a excepción de las ubicadas en la ciudad de Buenos Aires.

\section{Organismos internacionales y el traspaso de la educación normalista al nivel postsecundario y terciario (ca. 1940 - ca. 1960)}

\subsection{Reformas a la enseñanza normal chilena}

De manera parecida a la reforma Rothe en Argentina y, continuando lo que se había realizado en 1929 para las escuelas normales urbanas, un nuevo plan de estudios se implementó en 1944. De acuerdo con su contenido, el proceso de formación del maestro primario abarcaría dos ciclos: uno de Cultura General (compuesto por grupos de materias tales como Educación física y sanitaria, Educación científica, Educación artística, Educación técnica y Religión y moral), y otro de Cultura Profesional, el cual incluyó dos grupos de asignaturas, Ciencias auxiliares y filosofía, y Pedagogía. Respecto al proceso de titulación, el Reglamento de promoción y graduación de 1944 reafirmó las disposiciones de 1929 en lo relativo a la entrega del título de profesor. Al terminar sus estudios en la Normal, se entregaría una licencia, la cual permitiría ejercer. Luego de un año, los profesores licenciados debían solicitar una certificación final, cuya aprobación les otorgaría el título de profesor de educación primaria.

Sin embargo, el cambio más radical ocurrió a principios de la década de 1960 bajo la influencia de organismos internacionales como la Organización de las Naciones Unidas para la Educación, la Ciencia y la Cultura (Unesco), con la conformación de la Comisión de Planteamiento Integral de la Educación Chilena. Creada en 1962, la Comisión recomendó la aplicación de una nueva estructura del sistema escolar, que aumentaba la duración de la educación obligatoria de seis a ocho años, y reorientaba la enseñanza mediante la implementación de un moderno currículum escolar. En este nuevo escenario, la formación del profesorado tenía una importancia fundamental. Se recomendaba exigir que los candidatos hubiesen completado 
"doce años de estudio (9 del Ciclo de Educación General y 3 del Ciclo de Educación Media)" y que fueran sometidos a un cuidadoso proceso de selección.

A partir de lo anterior, entre 1964 y 1967 la enseñanza normal fue objeto de un proceso de modernización. En 1964 se aplicaron nuevos planes de estudio en las normales. En un contexto de planeamiento y reforma educacional ${ }^{4}$, se implementó un nuevo currículum en las normales, el cual estableció un plan de estudios de nueve años de duración. Esta modificación implicó la ascensión de la enseñanza normal a un nivel post secundario, el cual incluía una etapa de formación general de seis años de duración -equivalente a la enseñanza secundaria- y una etapa de formación profesional de tres años (Pérez Navarro, 2017). A esta última, los aspirantes podían ingresar directamente si contaban con la licencia de educación secundaria.

Estos cambios fueron complementados con el Programa de Desarrollo de la Enseñanza Normal, el cual contaba con la asistencia técnica de la Fundación Ford, de la Unesco y del Banco Mundial. Pero la oposición política en el Congreso Nacional y por parte de los gremios magisteriales bloquearon el programa (Cox y Gysling, 1990).

Finalmente, el sistema de escuelas normales fue suprimido en 1974, con posterioridad al Golpe de Estado llevado a cabo por las Fuerzas Armadas en septiembre de 1973 (Pérez Navarro, 2017). Si bien las autoridades militares argumentaron la suspensión del sistema de formación normalista debido a problemas relativos a financiamiento y calidad de la enseñanza 5 , las escuelas normales eran vistas por la Junta Militar como focos de subversión marxista (PIIE, 1984).

\subsection{El pasaje al nivel terciario no universitario en Argentina}

Igual que en Chile, la Argentina participó de las reuniones que convocaron Unesco y la Organización de los Estados Americanos (OEA) entre las décadas de 1950 y 1960, para hablar del "problema" de la formación docente en América Latina. En consonancia con estas deliberaciones, en Argentina también se realizaron encuentros para debatir sobre la enseñanza media en general, y las normales y la formación docente en particular. En ellos, hubo docentes que estaban de acuerdo con que la formación específica debía ser mayor de dos años.

\footnotetext{
${ }^{4}$ La reforma educacional de 1967 reestructuró la enseñanza primaria, sumando dos nuevos grados ( $7^{\circ}$ y $8^{\circ}$ año) El nuevo ciclo de primaria pasó a llamarse 'Educación Básica', y las carreras universitarias que formaban profesores se denominaron 'Pedagogía en Educación Básica'.

${ }^{5}$ De acuerdo con Núñez (2010), desde 1967 se comenzó a abandonar la formación normalista, lo cual quedó sellado en 1973 con la supresión de las escuelas normales.
} 
Mientras, entre 1956 y 1968, es decir, en solo 12 años se fundaron alrededor de 70 nuevas normales, buena parte de las cuales eran "Ciclos de Magisterio anexos", como ya mencionamos, el cuarto y quinto año (Rodríguez, 2019).

La dictadura de la "Revolución Argentina" (1966-1973) iniciada por el general Juan C. Onganía dio a conocer en diciembre de 1968 un Decreto que suprimió el Ciclo de Magisterio en los planes de estudio del nivel medio a partir de la iniciación del próximo período lectivo de 1969, durante el cual se cursaría por última vez el último año de dicho Ciclo. Por el Decreto 8051/68 se justificó la medida afirmando que "era conocido" el exceso de graduados en relación con el número de vacantes de cargos docentes, y que para mejorar su formación general había que exigir la aprobación de estudios completos de nivel medio como condición previa al ingreso a la carrera, y ello obligaba a situar la formación específicamente profesional en el nivel superior del sistema educativo. Se establecía que en los establecimientos donde se cursara el Ciclo de Magisterio, se podrían adoptar en su lugar, los siguientes planes: Bachillerato con Orientación Pedagógica; Bachillerato en Letras; Bachillerato en Ciencias Biológicas; Bachillerato en Ciencias Físico- Matemáticas; y Bachillerato con Orientación Agraria. En 1970 se abrió la inscripción para los nuevos Institutos Superiores de Formación Docente, ubicados en el nivel terciario. A diferencia de Chile, en esos años, las autoridades dictatoriales evitaron pasar la formación docente a la Universidad, porque consideraban que ese era el ámbito de la "subversión". En suma, el sistema público nacional terminó con alrededor de 174 normales nacionales, entre las que había 18 destinadas a formar al magisterio rural.

\section{Conclusiones}

En este artículo hemos analizado, en forma comparada, cómo se fue dando en Chile y Argentina el proceso de crecimiento de las normales desde principios del siglo XIX hasta los años de 1970. Específicamente, analizamos: a) los modelos extranjeros que influyeron en la enseñanza normal, b) el proceso de feminización del magisterio, y c) las reformas más importantes que fueron implementadas.

Respecto a los modelos extranjeros que influyeron en el normalismo, en la Argentina durante el siglo XIX hubo varios intentos de creación de normales de parte de las autoridades de la provincia de Buenos Aires: bajo influencia inglesa y siguiendo el método lancasteriano, se fundaron las dos primeras normales: en 1824 se creó la de mujeres (a iniciativa de la Sociedad de Beneficencia y subvencionada por el Estado, que cerró en los años de 1830) y en 1826 la de varones, que funcionó solo un par de años. En 1852 se diseñó la segunda Normal de varones emulando al modelo francés, pero ésta ni siquiera llegó a abrir sus puertas. En 1855 se fundó una nueva Normal de mujeres (también bajo la supervisión de la Sociedad 
de Beneficencia) que en 1873 tuvo una directora norteamericana al frente y fue clausurada en 1876. En 1865 se organizó la tercera Normal de varones que se mantuvo abierta por seis años y fue clausurada en 1871 por escasez de egresados. Luego de estos intentos poco exitosos de la provincia, siendo presidente Sarmiento, se comenzaron a fundar normales nacionales bajo el modelo norteamericano y con directores/as de ese país. En Chile, durante la segunda década del siglo XIX hubo creaciones bajo el modelo lancasteriano; mientras que, desde la fundación de las dos primeras normales a mediados de siglo, se impuso el modelo francés de formación docente. En tanto, hacia fines del siglo XIX, se reformó el sistema de enseñanza normal bajo el modelo alemán.

Sobre el proceso de feminización del magisterio, en Argentina se produjo a través de tres medidas que las autoridades tomaron en distintos momentos y que contribuyeron a profundizar esta tendencia: Sarmiento, cuando era funcionario de la provincia de Buenos Aires, alentó la presencia de mujeres en la profesión porque creía que ellas estaban "naturalmente" predispuestas para ocuparse de los/as niños/as pequeños/as y además, porque se les podía pagar menos, lo que hizo que hacia 1870 las maestras bonaerenses superaran en número a los maestros. Posteriormente, el gobierno nacional decidió que las normales de varones eran muy costosas en relación con el escaso número de egresados y ordenó cerrar entre 1900 y 1903, 12 de los 13 cursos de magisterio de varones que existían. Asimismo, en la ciudad de Buenos Aires se crearon 10 normales de mujeres entre 1874 a 1914, lo que aumentó exponencialmente el número de maestras. En Chile, las primeras normales fueron 12 de mujeres y 6 de varones, y ya desde mediados de la década de 1870, el número de maestras superó la cantidad de maestros. Si comparamos la creación de normales con otro tipo de establecimiento de nivel medio destinado a las mujeres, hemos visto que, en Chile, las autoridades desestimaron la fundación de más normales, y optaron por seguir abriendo Liceos Femeninos, superando en número a aquellas y llegando a ser más de 30 hacia 1970. En Argentina, al contrario, las élites políticas crearon muy pocos Liceos de Señoritas y priorizaron la fundación de normales que, hacia mediados del siglo XX eran mayoritariamente mixtas, pero con una matrícula casi enteramente femenina.

En referencia a las reformas, más allá de los numerosos cambios en los planes de estudio que hubo en Argentina, la reforma de 1941 cambió de raíz el sistema normalista, organizando un ciclo común de tres años para todas las escuelas medias y uno de dos años con la formación especializada. Producto de este proceso de fundación de normales, en la década de 1950, los dos países exhibían las tasas más altas de maestros/as titulados de América Latina. En simultáneo, en ambos países se hicieron sentir las influencias de los organismos internacionales -Unesco, OEA- que, en aras de la "modernización" de la formación docente, pedían que se agregaran más años a los planes de estudios o que se pasaran los cursos a otro nivel superior. En Argentina, en 1968, un gobierno dictatorial decidió por decreto 
trasladar la formación docente al nivel terciario. En tanto en Chile, hemos señalado que se produjo su pasaje al nivel post secundario en la misma década y, en el marco de la dictadura pinochetista, se eliminaron las normales y la formación docente pasó a la Universidad.

\section{Fuentes primarias}

Amunátegui, Domingo (1895), El sistema de Lancáster en Chile i en otros países sud americanos. Imprenta

Cervantes, Santiago.

Chile. Decreto 104. 8 de enero de 1964.

Chile. Decreto 19138. 27 de diciembre de 1962.

Chile. Decreto 332. 14 de febrero de 1928.

Chile. Escuela Normal de Preceptoras de Santiago. Decreto. 5 de enero de 1854.

Chile. Escuela Normal de Preceptores de Santiago. Decreto. 18 de enero de 1842.

Chile. Ley 5100. 13 de noviembre de 1929.

El Monitor, № 505, 1915.

Fernández, Juan (1903), Antecedentes sobre enseñanza secundaria y normal en la República Argentina,

Taller Tipográfico de la Penitenciaría Nacional, Buenos Aires.

Lucero, Jesús (1951), Reseña histórica de la Escuela Normal Superior Juan Pascual Pringles en el 75 Aniversario de su fundación. 1876-1951, Universidad Nacional de Cuyo, San Luis.

Ministerio de Educación Pública (1945), Sarmiento. Director de la Escuela Normal. 1842-1845, Imprenta Universitaria, Santiago.

Núñez, José Abelardo (1883), Organización de escuelas normales, Imprenta de la Librería Americana, Santiago.

Oficina Central de Estadística (1910), Anuario Estadístico de la República de Chile, Oficina Central de Estadística, Santiago.

Portnoy, Antonio (1937), La Instrucción primaria desde 1810 hasta la sanción de la ley 1420, CNE, Buenos Aires.

Ramos, Juan (1910), Historia de la Instrucción Primaria en la República Argentina, 1810-1910. Tomos I y II,

Peuser, Buenos Aires.

Revista de Educación, № 100, 1959.

Salvadores, Antonino (1941), La instrucción primaria desde 1810 hasta la sanción de la Ley 1420, Consejo Nacional de Educación, Buenos Aires

Sarmiento, Domingo Faustino (1954), Obras Completas de Sarmiento XLIV. Informes sobre educación, Editorial Luz del Día, Buenos Aires.

- (2011 [1849]), Educación Popular, UNIPE, Buenos Aires.

\section{Referencias hemerográficas}

Fiorucci, Flavia (2014), "Maestros para el sistema de educación pública. La fundación de escuelas normales en Argentina (1890-1930)" en Revista Mexicana de Historia de la Educación, Sociedad Mexicana de Historia de la Educación, México, vol. 2, núm. 3, pp. 25-45.

Mansilla, Juan (2018), "Influencia alemana en la reforma de las Escuelas normales de Preceptores y Preceptoras en el centro sur de Chile, 1883-1920" en Revista Historia de la Educación Latinoamericana, vol. 20, núm. 3, pp. 189-209. 
Reyes, Leonora (2010), "Movimiento educacional, crisis educativa y reforma de 1928", en Docencia, núm. 40, pp. 40-49.

Núñez, Iván (2010), "Escuelas normales: una historia larga y sorprendente. Chile (1842-1973)" en Pensamiento Educativo, vol. 46-47, pp. 133-150.

Rodríguez, Laura Graciela (2021), "Maestros y maestras y la cuestión de género: planes de estudio, salarios y feminización (Argentina, 1870-1914)" en Descentrada. Revista interdisciplinaria de feminismos y género, núm. 1, pp. 1-17.

-(2020), "Las Escuelas Normales creadas para formar maestros/as rurales (Argentina, 1903-1952)" en Mundo Agrario, vol. 21, núm. 47, pp. 1-25.

(2019), "Cien años de normalismo en Argentina (1870-1970). Apuntes sobre una burocracia destinada a la formación de docentes" en Ciencia, Docencia y Tecnología, vol. 30, núm. 59, pp. 200-235.

Sanhueza, Marcelo (2013), "El viaje a París de Domingo Faustino Sarmiento y Benjamín Vicuña Mackenna: modernidad y experiencia urbana de dos flâneurs hispanoamericanos" en Universum, Vol. 1, núm. 28, pp. 203-229.

\section{Referencias bibliográficas}

Alliaud, Andrea (2007), Los maestros y su historia. Los orígenes del magisterio argentino, Granica, Buenos Aires.

Ávalos, Beatrice (2003), La formación docente inicial en Chile, Unesco, Santiago.

Conejeros, Juan Pablo (1999), La influencia cultural francesa en la educación chilena, 1840-1880, Universidad Católica Silva Henríquez, Santiago.

-(2015), "De la francomanía al Embrujo alemán. Alcances en torno al rol de los agentes mediatizadores en el proceso de transferencia cultural alemana en la educación chilena (1880-1910)" en Benjamín Silva (Comp.), Historia social de la educación chilena. Tomo I: Instalación, auge y crisis de la reforma alemana 1880 a 1920. Agentes escolares, Ediciones UTEM, Santiago, pp. 36-67.

Cox, Cristián y Jacqueline Gysling (1990), La formación del profesorado en Chile (1842- 1987), CIDE, Santiago.

Egaña, María Loreto (1994), Espacio escolar y actores en la educación primaria popular en el siglo XIX en Chile, PIIE, Santiago.

Egaña, María Loreto (2000), La educación primaria popular en el siglo XIX en Chile: una práctica de política estatal, DIBAM, Santiago.

Egaña, María Loreto, Iván Núñez y Cecilia Salinas (2003), La educación primaria en Chile: 1860-1930. Una aventura de niñas y maestras, LOM-PIIE, Santiago.

Labarca, Amanda (1939), Historia de la enseñanza en Chile, Imprenta Universitaria, Santiago.

Lionetti, Lucía (2007), La misión política de la escuela pública: la formación de los ciudadanos en Argentina, 1870-1916, Editorial Miño y Dávila, Buenos Aires.

Mancilla, Arturo (2005), Antecedentes para una historia de la educación primaria en Chile. Siglos XIX y comienzos del XX, Tesis para optar al grado de Magíster en Historia, Universidad de Chile, Santiago.

Munizaga, Roberto (1990), Nuevos ensayos sobre educación, Imprenta Universitaria, Santiago.

Muñoz Hermosilla, José María (1918), Historia elemental de la pedagojía chilena, Casa Editorial Minerva, Santiago.

Newland, Carlos (1992), Buenos Aires no es pampa. La educación elemental porteña, 1820-1860, Grupo Editor Latinoamericano, Buenos Aires. 
Orellana, María Isabel (2007), Educación: improntas de mujer, DIBAM/Museo de la Educación Gabriela Mistral, Santiago.

Peña, Macarena (2000), Hijas amadas de la patria: historia de la Escuela Normal de Preceptoras de Santiago, 1854-1883, Tesis para optar al grado de licenciada en historia, Pontificia Universidad Católica de Chile, Santiago.

Pérez Navarro, Camila (2017), Escuelas normales en Chile: una mirada a sus últimos intentos de modernización y a su proceso de cierre (1961-1974), Colecciones Digitales/Subdirección de Investigación, DIBAM

Pérez Navarro, Camila (2020), Iniciativas, prácticas y límites de la experimentación pedagógica en la historia de la educación chilena (1927-1953), Colecciones Digitales/Museo de la Educación Gabriela Mistral.

PIIE (1984), Las transformaciones educacionales bajo el régimen militar, PIIE, Santiago.

Reyes, Leonora (2014), La escuela en nuestras manos. Las experiencias educativas de la Asociación General de Profesores y la Federación Obrera de Chile (1931-1932), Quimantú, Santiago.

Rojas Flores, Jorge (2010), Historia de la infancia en el Chile republicano, 1810-2010, Ocho Libros, Santiago.

Serrano, Sol (1993), Universidad y nación. Chile en el siglo XIX, Editorial Universitaria, Santiago. - Macarena Ponce de León y Francisca Rengifo (2013), Historia de la Educación en Chile. Tomo II: La educación nacional (1880-1930), Taurus, Santiago.

Soto Roa, Fredy (2013), Historia de la Educación Chilena, Ediciones Universidad Central, Santiago. Vial, Gonzalo (1996), Historia de Chile, 1891-1973, Zig-Zag, Santiago. 